\title{
Highly pressurized partially miscible liquid-liquid flow in a micro-T-junction. II. Theoretical justifications and modeling
}

\author{
Ning Qin, John Z. Wen, and Carolyn L. Ren* \\ Department of Mechanical and Mechatronics Engineering, University of Waterloo, Waterloo, Ontario, Canada N2L 3G1
}

(Received 12 October 2016; published 24 April 2017)

\begin{abstract}
This is the second part of a two-part study on a partially miscible liquid-liquid flow (carbon dioxide and deionized water) that is highly pressurized and confined in a microfluidic T-junction. In the first part of this study, we reported experimental observations of the development of flow regimes under various flow conditions and the quantitative characteristics of the drop flow including the drop length, after-generation drop speed, and periodic spacing development between an emerging drop and the newly produced one. Here in part II we provide theoretical justifications to our quantitative studies on the drop flow by considering (1) $\mathrm{CO}_{2}$ hydration at the interface with water, (2) the diffusion-controlled dissolution of $\mathrm{CO}_{2}$ molecules in water, and (3) the diffusion distance of the dissolved $\mathrm{CO}_{2}$ molecules. Our analyses show that (1) the $\mathrm{CO}_{2}$ hydration at the interface is overall negligible, (2) a saturation scenario of the dissolved $\mathrm{CO}_{2}$ molecules in the vicinity of the interface will not be reached within the contact time between the two fluids, and (3) molecular diffusion does play a role in transferring the dissolved molecules, but the diffusion distance is very limited compared with the channel geometry. In addition, mathematical models for the drop length and the drop spacing are developed based on the observations in part I, and their predictions are compared to our experimental results.
\end{abstract}

DOI: 10.1103/PhysRevE.95.043111

\section{INTRODUCTION}

In the first paper, we reported the experimental observations on partially miscible liquid-liquid flows that was highly pressurized and confined in a microfluidic T-junction, where liquid carbon dioxide and water were used as the dispersed and the continuous liquids, respectively. Under the flow conditions (different flow rate ratios and capillary numbers) probed, drop flow and coflow were identified as the two main flow patterns, where the "drop flow" was a designation covering the squeezing, transitional, and dripping regime. Moreover, the squeezing stage was found in our study as sort of "elongatingsqueezing" because of the significant "dragging" effect due to the viscous forces exerted on the interface even during the commonly acknowledged, interfacial tension-dominated squeezing regime. The "flowrate-controlled" mechanism [1,2] was suggested to interpret the elongating-squeezing regime of the drop breakup. However, our scaling formulations from the experimental results indicated a magnified effect of the flow rate contrast upon the final sizes of the produced liquid $\mathrm{CO}_{2}$ drops (i.e., the factor $\alpha$ in the formulation reported by Garstecki et al. is larger than as expected [1]). There exists a transitional capillary number $\mathrm{Ca}_{c} \sim 1 \times 10^{-2}$, as confirmed quantitatively, when the drop formation transits from the elongating-squeezing to the dripping regime. Focusing on the drop flows, we measured the speed values of the drops following generations as they started to flow in the main channel and characterized the periodical development of drop spacing between the emerging drop and the newly produced one.

In this second paper we first provide theoretical justifications to our quantitative observations on the drop flow from the relevant aspects of the mass transfer of the $\mathrm{CO}_{2}$ molecules that are mainly present in the vicinity of the interface between the bulk $\mathrm{CO}_{2}$ and the water stream. The relevant

\footnotetext{
*c3ren@uwaterloo.ca
}

mass transfer mechanisms being investigated include (1) $\mathrm{CO}_{2}$ hydration at the interface with water, (2) the diffusioncontrolled dissolution of $\mathrm{CO}_{2}$ molecules in water, and (3) the diffusion distance of the dissolved $\mathrm{CO}_{2}$ molecules.

Focusing on the drop length and the drop spacing, two mathematical models are provided in this paper based on the experimental results reported in part I. The model for the drop length accounts for the effect of the "elongating time" added to the "necking time" on the final sizes of the generated drops, which elucidates the factor $A$ (much larger than 1 ) for our cases in the formulation

$$
L / W \sim 1+A\left(Q_{\mathrm{H}_{2} \mathrm{O}} / Q_{\mathrm{CO}_{2}}\right)^{B},
$$

where $L$ and $W$ are the drop length $(\mu \mathrm{m})$ and the channel width $(150 \mu \mathrm{m})$, respectively; $Q_{\mathrm{H}_{2} \mathrm{O}}$ and $Q_{\mathrm{LCO}_{2}}$ are the volumetric flow rates $(\mu \mathrm{l} / \mathrm{min})$ of water and liquid $\mathrm{CO}_{2}$; and $A$ and $B$ are fitting factors from the experiments. Based on the drop speed differences in the stages of one period of the drop generation, a linear mathematical model is built to predict the drop spacing developments between an emerging drop and the newly produced one within the stagnating and filling stage and the elongating and squeezing stage of one drop generation period. (The truncating stage is very short comparatively and therefore is not considered.)

\section{THEORETICAL JUSTIFICATIONS}

When it comes to the two-phase microflows concerning fully miscible and/or partially miscible fluids, we meet mostly a diffusive-interface problem especially when we eye the interfacial phenomenon, such as the hydrodynamics occurring near contact lines or breakup and coalescence related to fluid jets as well as droplets [3]. The instance of using liquid $\mathrm{CO}_{2}$ and water as the dispersed and the continuous fluid in the micro-T-junction is very likely one of those problems. This statement is further verified by others' experimental observations $[4,5]$ where the interface separating the bulk $\mathrm{CO}_{2}$ from 
the water stream in the microchannels is featured by a dark ring enclosing the $\mathrm{CO}_{2}$ drop under bright-field visualizations. We believe that there exists a dissolution-diffusional film, instead of the "sharp interface," in which the concentration gradient of dissolved $\mathrm{CO}_{2}$ molecules leads to a gradient of the density that further results in different refraction coefficients. Therefore, to justify our previous experimental results of the drop flows, it is very necessary to take the mass transport of the $\mathrm{CO}_{2}$ molecules (into water from the $100 \%$ interface) into account and to estimate how much $\mathrm{CO}_{2}$ dissolves and how far the dissolved $\mathrm{CO}_{2}$ molecules can diffuse into the water stream. Prior to a discussion of the diffusion-controlled dissolution, the hydration of the dissolved $\mathrm{CO}_{2}$ molecules is analyzed.

\section{A. Interfacial dissolution of $\mathrm{CO}_{2}$ in water within the T-junction}

\section{Hydration of the dissolved $\mathrm{CO}_{2}$ molecules}

The hydration of $\mathrm{CO}_{2}$ occurs when it dissolves in water $(\mathrm{pH} \approx 5.93)$ and produces carbonic acid, as formulated by the reaction at chemical equilibrium

$$
\mathrm{CO}_{2}(a q)+\mathrm{H}_{2} \mathrm{O} \rightleftharpoons \mathrm{H}_{2} \mathrm{CO}_{3}(a q) .
$$

The equilibrium constant $K_{\mathrm{CO}_{2}}$, as defined by the ratio of the reaction rate constant $\left(k_{f}\right)$ of the forward reaction over that $\left(k_{r}\right)$ of the reverse reaction under equilibrium $\left\{k_{f} / k_{r}\right.$, which also equals the concentration ratio $\left[\mathrm{H}_{2} \mathrm{CO}_{3}\right] /\left(\left[\mathrm{CO}_{2}\right] \times\right.$ $\left.\left[\mathrm{H}_{2} \mathrm{O}\right]\right)$ at equilibrium, where the square bracket denotes the concentrations in the unit of mol/liter; however, this reaction is generally a pseudo-first-order reaction because of a constant $\left[\mathrm{H}_{2} \mathrm{O}\right]\left(\left[\mathrm{H}_{2} \mathrm{O}\right]\right.$ can thus be omitted out of the denominator), thus $\left.k_{f} / k_{r}=\left[\mathrm{H}_{2} \mathrm{CO}_{3}\right] /\left[\mathrm{CO}_{2}\right]\right\}$ indicates how much $\mathrm{CO}_{2}$ can be hydrated into carbonic acid. At $25^{\circ} \mathrm{C}, K_{\mathrm{CO}_{2}} \approx 1.7 \times 10^{-3}$ (unit:1) for the above reaction indicates the vast majority of $\mathrm{CO}_{2}$ exists as molecular $\mathrm{CO}_{2}$ rather than $\mathrm{H}_{2} \mathrm{CO}_{3}$ in water [6]. The other fact is that the forward process is much slower than the reverse at room temperature $\left(k_{f} \approx 0.035 \mathrm{~s}^{-1}\right.$ versus $k_{r} \approx 20.6 \mathrm{~s}^{-1}$ for the first-order reaction with respect to $\mathrm{CO}_{2}$ and $\left.\mathrm{H}_{2} \mathrm{CO}_{3}[7,8]\right)$. Given by the time scale of the $\mathrm{CO}_{2}$ drop generations at the T-junction (see Table II in paper I but without considering case 13 and 21), the hydration of dissolved $\mathrm{CO}_{2}$ molecules has not reached equilibrium.

When the concentration of $\mathrm{H}_{2} \mathrm{CO}_{3}$ is relatively low in the aqueous solution, an overall reaction including the dissociation of $\mathrm{H}_{2} \mathrm{CO}_{3}$ for producing bicarbonate $\mathrm{HCO}_{3}{ }^{-}$is usually introduced to replace Eq. (2):

$$
\mathrm{CO}_{2}+\mathrm{H}_{2} \mathrm{O} \rightleftharpoons \mathrm{HCO}_{3}{ }^{-}+\mathrm{H}^{+} .
$$

Note that the $\mathrm{CO}_{2}$ in the above reaction includes both the aqueous $\mathrm{CO}_{2}$ and the hydrated species $\left(\mathrm{H}_{2} \mathrm{CO}_{3}\right)$. The equilibrium constant $\left(K_{\mathrm{CO}_{2}}^{\prime}\right)$ of this reaction can be expressed as

$$
K_{\mathrm{CO}_{2}}^{\prime}=k_{f}^{\prime} / k_{r}^{\prime}=\left[\mathrm{HCO}_{3}^{-}\right]\left[\mathrm{H}^{+}\right] /\left(\left[\mathrm{CO}_{2}\right]\left[\mathrm{H}_{2} \mathrm{O}\right]\right) .
$$

The value of $K_{\mathrm{CO}_{2}}^{\prime}$ at $25^{\circ} \mathrm{C}$ and a very low ionic strength $(\sim 0)$ is $4.45 \times 10^{-7}$ [9]; or, $p K_{a}\left(p K_{a}=-\log _{10} K_{a}\right)$ is 6.35 as an apparent dissociation constant of nominal $\mathrm{H}_{2} \mathrm{CO}_{3}$ which includes both $\mathrm{CO}_{2}(\mathrm{aq})$ and the rare $\mathrm{H}_{2} \mathrm{CO}_{3}$ [6]. In fact, the $p K_{a}$ of carbonic acid (purely $\mathrm{H}_{2} \mathrm{CO}_{3}$ ) is $\sim 3.6$ at $25^{\circ} \mathrm{C}$ [6]. Furthermore, bicarbonate may dissociate into hydrogen ion
$\left(\mathrm{H}^{+}\right)$and carbonate ions $\left(\mathrm{CO}_{3}{ }^{-}\right)$by the reaction

$$
\mathrm{HCO}_{3}{ }^{-} \rightleftharpoons \mathrm{CO}_{3}{ }^{-}+\mathrm{H}^{+},
$$

which is characterized by a second equilibrium constant $K_{\mathrm{HCO} 3}{ }^{-}$defined as

$$
K_{\mathrm{HCO}_{3}{ }^{-}}=\left[\mathrm{CO}_{3}{ }^{2-}\right]\left[\mathrm{H}^{+}\right] /\left[\mathrm{HCO}_{3}{ }^{-}\right] .
$$

Comparatively, $K_{\mathrm{HCO}_{3}{ }^{-}}$has a value of $4.84 \times$ $10^{-11}$ (mol/liter), and thus the dissociation constant $p K_{a}$ of $\mathrm{HCO}_{3}{ }^{-}$is 10.3 [9]. Due to its much lower dissociation constant $\left(p K_{a} \approx 14\right.$ at $25^{\circ} \mathrm{C}$ ) [10], water's self-ionization is beyond the scope of our discussion. In a nutshell, the hydration of dissolved $\mathrm{CO}_{2}$ at the interface shows an overall negligible effect to the total dissolved $\mathrm{CO}_{2}$ molecules, and they are still vastly present as $\mathrm{CO}_{2}$ molecules in the aqueous solution, given the time scale in the micro-T-junction.

\section{Theoretical estimate of diffusion-controlled dissolution}

$\mathrm{CO}_{2}$ molecules, after their dissolution in water [i.e., dissolved $\mathrm{CO}_{2}(\mathrm{aq})$ molecules], can be transported from the bulk $\mathrm{CO}_{2}$ to water, which is mainly attributed to the diffusion of the $\mathrm{CO}_{2}(\mathrm{aq})$ molecules under a certain concentration gradient. In general, several mechanisms should be considered when discussing mass transfer, which mainly include hydrodynamic dispersion, convection, and diffusion. Considering a mixing zone with a concentration gradient of the sample of interest in a pressure-driven microchannel flow, velocity profile is parabolic type which causes distortion to the mixing zone and must be considered in evaluating the mass transfer in this region. However, for the mixing zone shown in Figure 1, which is a small region of the entire interface between the dispersed and continuous phase in the $x$ - $y$ plane, hydrodynamic dispersion is negligible. The curved interface is meant to illustrate the impact of the interfacial tension between the $\mathrm{CO}_{2}$ drop and the thin water film that exists between the $\mathrm{CO}_{2}$ drop and channel walls in the $z$ direction (top and bottom channel walls). First, the velocity component in the $y$ direction (channel height direction) is small, which is mainly induced by the vortices inside the droplets. Therefore, the hydrodynamic distortion to the mixing zone caused by the velocity component in the $y$ direction is negligible. Second, the velocity component in the $x$ direction is also small. During the filling and stagnation stage, there exists a velocity component in the $x$ direction which is much smaller than that in the $z$ direction. When the drop formation evolves from the filling to the elongating and squeezing stage, which is the longest stage among the three stages, the velocity component in the $x$ direction is approaching zero because the interface is almost parallel to the vertical channel walls. Therefore, its overall impact on hydrodynamic dispersion is negligible. Third, the possible distortion to the mixing zone could be caused by the shear motion over the interface, which is induced by the difference of the velocity component in the $z$ direction between the continuous and dispersed phases. However, this hydrodynamic distortion is also negligible if considering the entire drop formation period. This study operates in the squeezing regime, and in the longest stage, the elongating and squeezing stage, the continuous phase upstream is almost completely blocked by the $\mathrm{CO}_{2}$ drop, which almost touches the channel walls. 
(a)
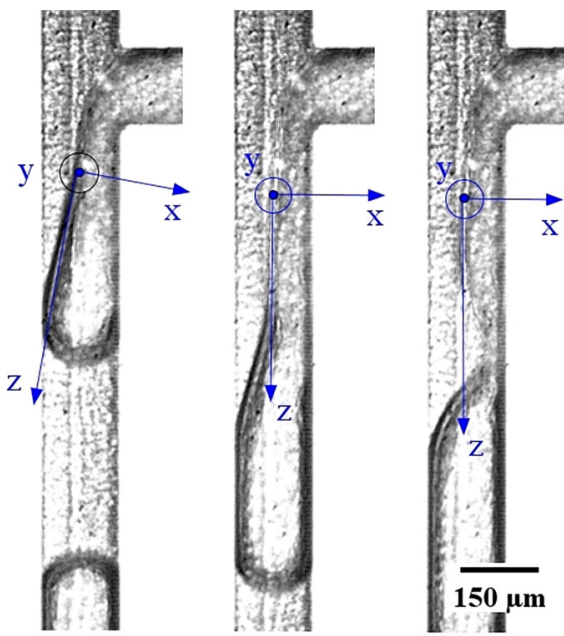

(b)

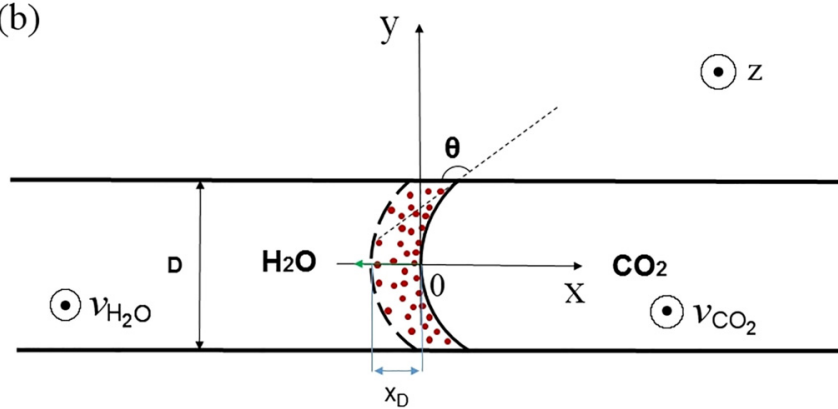

FIG. 1. (a) Indications of the Cartesian coordinates ( $x$ : perpendicular to the liquid $\mathrm{CO}_{2}$ stream; $y$ : tangential to the interface and in the channel depth direction; $z$ : tangential to the interface and in the flow direction; origin: one point-of-interest on interface at a half channel depth), scale bar: $150 \mu \mathrm{m}$. (b) Schematic of the transport of the dissolved $\mathrm{CO}_{2}$ molecules from the interface (solid line) into water driven by dissolution and diffusion (in the $x-y$ plane). The region outlined by a solid line and a dash line represents a diffusive film of the $\mathrm{CO}_{2}$ molecules. Note that this diffusive film is enlarged for easy viewing and is actually very thin compared to the channel depth $D\left(X_{D} / D \sim 10^{-2}\right.$, where $X_{D}$ is the thickness of the diffusion film.). The schematic shows a cross-sectional view of the two phases separated by two interfaces, one (the solid line) is between $\mathrm{CO}_{2}$ and the $\mathrm{CO}_{2}$ aqueous solution, and the other (the dash line) is a hypothetical one between pure water and the $\mathrm{CO}_{2}$ aqueous solution where $\mathrm{CO}_{2}$ concentration is nonzero but approaching zero.

This results in a negligible shear motion over the interface and thus negligible hydrodynamic distortion. During the first short filling stage, the shear motion is appreciable. However, its effect in distorting the mixing zone is reduced to a certain extent by the interfacial tension that tends to hold the interface in shape. Therefore, overall the hydrodynamic dispersion caused by the shear motion can be neglected.

The $\mathrm{CO}_{2}$ transport over the mixing zone region due to convection is mainly influenced by the velocity component in the $x$ direction because the velocity in the $z$ direction is tangential to the interface. As discussed above, the velocity component in the $x$ direction is very small during the filling stage and almost approaches to zero during the long elongating and squeezing stage. Therefore the mass transfer of $\mathrm{CO}_{2}$ over the mixing zone is mainly dominant by diffusion.
The dissolution and diffusion of the $\mathrm{CO}_{2}$ molecules is schematically described in Fig. 1. Due to the unsteady interface in terms of its location and shape, we assume the following:

(1) Water is an isotropic and homogeneous solvent

(2) The diffusion coefficient of $\mathrm{CO}_{2}$ molecules into water, $D_{C D}$, is a constant

(3) The effect of the finite water film (thickness $\approx 2 \%$ $\times$ hydraulic diameter [4]), which exists between $\mathrm{CO}_{2}$ and the channel walls due to wettability, on $\mathrm{CO}_{2}$ dissolution and diffusion is negligible, and thus we only consider the transport process across the diffusive film (shown in Fig. 1)

(4) Mass transport is one-dimensional in the direction perpendicular to the interface ( $x$ direction in the Fig. 1), in other words, we focus on the $x-y$ plane

(5) Quasi-steady state is achieved, which is rational especially in the "elongating and squeezing" stage, the generally longest stage compared to the other two stages, and therefore the location of the interface is constant relative to the channel wall; and the hydrodynamic dispersion on the $x$ axis may approach zero

(6) Other than the interface (specifically, the solid line in Fig. 1) as a boundary, the water-side channel wall is another one in the $x$ direction.

For this one-dimensional model under a quasi-steady state (on the negative " $x$ " direction), the dissolution of $\mathrm{CO}_{2}$ molecules at the interface (solid line in Fig. 1) and the diffusion of the dissolved $\mathrm{CO}_{2}(a q)$ molecules in water can be described by the following two equations:

$$
\begin{aligned}
\left.\frac{d C}{d t}\right|_{x \sim 0} & =K_{C D} A\left(C_{s}-C\right), \\
J_{x} & =-D_{C D} \frac{d C}{d x},
\end{aligned}
$$

where $C$ is the concentration of the dissolved $\mathrm{CO}_{2}$ in water at time $t\left(0<t<t_{0}\right), C_{s}$ is the solubility of $\mathrm{CO}_{2}$ in water at a given pressure and temperature, $K_{C D}$ is a constant with a unit of $1 /\left(\mathrm{m}^{2} \cdot \mathrm{s}\right)$, and $A$ is the effective dissolution area (i.e., the area of the concave interface). Equation (8) is Fick's first law of diffusion, where $J_{x}\left[\mathrm{~mol} /\left(\mathrm{m}^{2} \times \mathrm{s}\right)\right]$ is the diffusion molar flux of the dissolved $\mathrm{CO}_{2}$ molecules per square meter per second and it is related to the concentration gradient. Note that we omit the convective molar flux in this binary mixture caused by the molar average velocity in view of the very small molar fraction $\left[C /\left(C+C_{\mathrm{H}_{2} \mathrm{O}}\right) \leqslant 2 \times 10^{-3}\right]$ of $\mathrm{CO}_{2}$ in the mixture of $\mathrm{CO}_{2}$ and water.

Integration of Eq. (7) with Eq. (8) at $x \sim 0$ with time approaching infinite $\left(X_{D} \sim W / 2\right.$ as $\left.t \sim \infty\right)$ leads to

$$
\left.\frac{d C}{d t}\right|_{x \sim 0}=K_{C D} A \frac{W}{2} \frac{J_{0}}{D_{C D}} .
$$

If we further introduce $V$ as the final dissolution volume, Eq. (9) can be revised as

$$
\left.\frac{1}{V} \frac{d(V C)}{d t}\right|_{x \sim 0}=K_{C D} A \frac{W}{2} \frac{J_{0}}{D_{C D}},
$$

in which $d(V C) / d t$ at $x \sim 0$ offsets $J_{o} \cdot A(\mathrm{~mol} / \mathrm{s})$ and yields the factor $K_{C D}$ :

$$
K_{C D}=\frac{D_{C D}}{V W / 2}
$$


Thus Eq. (7) can be rewritten as

$$
\left.\frac{d C}{d t}\right|_{x \sim 0}=\frac{D_{C D} A}{V W / 2}\left(C_{s}-C\right) .
$$

Equation (12) describes the dissolution of $\mathrm{CO}_{2}$ molecules at the interface in terms of the concentration variations over a sufficiently long period, which is dominated by diffusion. The integration of this equation leads to

$$
C_{x \sim 0}=C_{s}\left(1-e^{-\frac{D_{C D} A}{V W / 2} t}\right) .
$$

Based on an approximation of $V \sim(A \cdot W / 2)$, Eq. (13) may be simplified as

$$
C_{x \sim 0}=C_{s}\left(1-e^{-\frac{4 D_{C D}}{W^{2}} t}\right) .
$$

The diffusion coefficient of $\mathrm{CO}_{2}$ molecules into water, $D_{C D}$, at $298 \mathrm{~K}$ can be estimated by the Stokes-Einstein relation [11],

$$
D_{C D}=\frac{k_{B} T}{6 \pi \eta r},
$$

where $k_{B}, \eta$, and $r$ are the Boltzmann constant $\left(k_{B}=\right.$ $\left.1.38 \times 10^{-23} \mathrm{~J} / \mathrm{K}\right)$, the dynamic viscosity $(\eta=890 \mu \mathrm{Pa} \cdot \mathrm{s}$ at $298 \mathrm{~K}$ ) of water and the kinetic radius of the hypothetical sphere-shaped $\mathrm{CO}_{2}$ molecules $\left(r=1.65 \AA=1.65 \times 10^{-10} \mathrm{~m}\right.$ according to Ref. [12]), respectively. Therefore, $D_{C D}$ has an approximate value of $1.5 \times 10^{-9} \mathrm{~m}^{2} / \mathrm{s}$, and Eq. (14) can be specifically written for our case as

$$
C_{x \sim 0}=C_{s}\left(1-e^{-\frac{4}{15} t}\right) .
$$

Moreover, $C_{s}$ at the applied pressure and temperature condition in our study has a value of $1.267 \mathrm{~mol} / \mathrm{liter}$ or so [13,14] where the molar fraction of $\mathrm{CO}_{2}$ is approximately 0.0247 mole per 1 mole water. As a result, Eq. (16) can be further specified as

$$
C_{x \sim 0}(\mathrm{~mol} / \text { liter })=1.267\left(1-e^{-\frac{4}{15} t}\right) .
$$

In view of the time durations in Table II in part I, it is too fast to render a saturation scenario of the dissolved $\mathrm{CO}_{2}$ at the interface, though a large number $\left(10^{-4} N_{A}, N_{A}\right.$ is the Avogadro number.) of $\mathrm{CO}_{2}$ molecules at least conceptually exist in the aqueous solution near the interface.

\section{B. Molecular diffusion of dissolved $\mathrm{CO}_{2}$ molecules in water}

As discussed in the previous section, the interface between liquid $\mathrm{CO}_{2}$ and water within the T-junction during the second stage and the third stage of one period is featured by a clear upper section and a shading lower section. The clear section, as observed, barely moves relative to the shading section due to a compromise of the capillary pressure in squeezing the $\mathrm{CO}_{2}$ stream. This section of the interface is considered to be quasisteady and diffusion becomes the only transport mechanism of the dissolved $\mathrm{CO}_{2}$ molecules. To evaluate how far the $\mathrm{CO}_{2}$ molecules can be transported into water, namely, the diffusion distance, an appropriate solution of Fick's second law may be required [15]. The diffusion equation for one dimensional model at a nonsteady state has been defined by Fick's second law [16], hence,

$$
\frac{\partial C}{\partial t}=D_{C D} \frac{\partial^{2} C}{\partial x^{2}}
$$

This partial differential equation can be solved based on a constructed function $\mathrm{y}=\frac{x}{\sqrt{4 D_{C D} t}}$ and rearranged as an ordinary differential equation of $C$ with respect to $y$. Here we provide only the final expression of the solution [see Eq. (19)] and the detailed deductions can be found in literature $[17,18]$ :

$$
\int d C=\int B e^{-y^{2}} d y
$$

where $B$ is a constant resulted from the integration. To solve the above integrals, the following boundary conditions need to be used:

$$
\begin{aligned}
C_{x} & =C_{0} \text { at } y=0(x=0, t>0) \\
& =0, \text { at } y=\infty(x=\infty, t=0) .
\end{aligned}
$$

Therefore, we have

$$
\int_{c_{0}}^{0} d c=B \int_{0}^{\infty} \mathrm{e}^{-y^{2}} d y .
$$

Based on the property of error function $\operatorname{erf}(y)$ and complementary error function $\operatorname{erfc}(y)$, we have

$$
\begin{aligned}
\operatorname{erf}(y) & =\frac{2}{\sqrt{\pi}} \int_{0}^{y} \mathrm{e}^{-\xi^{2}} d \xi \\
\operatorname{erfc}(y) & =1-\operatorname{erf}(y)=\frac{2}{\sqrt{\pi}} \int_{y}^{\infty} \mathrm{e}^{-\xi^{2}} d \xi .
\end{aligned}
$$

Let $y=0$ in Eqs. (22a) and (22b), we obtain

$$
\int_{0}^{\infty} \mathrm{e}^{-y^{2}} d y=\frac{\sqrt{\pi}}{2} .
$$

Substituting Eq. (23) into Eq. (21) gives

$$
B=\frac{-2 c_{0}}{\sqrt{\pi}} \text {. }
$$

Substituting $B$ back to Eq. (19) yields

$$
\int d C=\frac{-2 c_{0}}{\sqrt{\pi}} \int \mathrm{e}^{-y^{2}} d y .
$$

Solving the integrals from $y=0$ to $y=\frac{x}{\sqrt{4 D_{C D} t}}$ on the two sides of Eq. (25), we have

$$
\int_{c_{0}}^{c_{x}} d c=\frac{-2 c_{0}}{\sqrt{\pi}} \int_{0}^{y=\frac{x}{\sqrt{4 D_{C D}}}} \mathrm{e}^{-\xi^{2}} d \xi
$$

or

$$
c_{x}-c_{0}=\left(-c_{0}\right) \operatorname{erf}(y) .
$$

The error function $\operatorname{erf}(y)$ can be expanded using Taylor series as

$$
\begin{aligned}
\operatorname{erf}(y) & =\frac{2}{\sqrt{\pi}} \sum_{n=0}^{\infty} \frac{(-1)^{n}(y)^{2 n+1}}{n !(2 n+1)} \\
& =\frac{2}{\sqrt{\pi}}\left(y-\frac{y^{3}}{3}+\frac{y^{5}}{10}-\frac{y^{7}}{42}+\cdots\right) .
\end{aligned}
$$

The first term on the right side of Eq. (27) is applied to approximate the error function, and hence,

$$
\operatorname{erf}(y) \sim \frac{2}{\sqrt{\pi}} y=\frac{2}{\sqrt{\pi}} \frac{\mathrm{x}}{\sqrt{4 D_{C D} t}}=\frac{\mathrm{x}}{\sqrt{\pi D_{C D} t}} .
$$


Substituting Eq. (28) into Eq. (26b) yields

$$
c_{x}=c_{0}\left(1-\frac{x}{\sqrt{\pi D_{C D} t}}\right) .
$$

$C_{x}$ is the concentration of the dissolved $\mathrm{CO}_{2}$ molecules at a diffusion distance $x$ from the interface (in Fig. 1) and let $c_{x} \approx 0$ be a critical concentration, then we have

$$
|x| \approx \sqrt{\pi D_{C D} t}
$$

where $t$ is the diffusion time. It is obvious that the absolute value of the diffusion distance $x$ mainly depends on the diffusion time $t$ since diffusion coefficient is a constant $\left[O\left(10^{-9}\right)\right]$. However, $t$ is uneasy to be exactly quantified because the hydrostatic state of one specific point on the clear section lasts for only a limited time $(t)$ out of one period $t_{0}$. Diffusion time $t$ can be written as

$$
t=a t_{0}, \quad 0<a<1,
$$

where $a$ is a fractional number. Here $a$ varies among different cases with different $Q_{\mathrm{H}_{2} \mathrm{O}} / Q_{L \mathrm{CO}_{2}}$; even under the same case, it also varies among various locations on the clear section. Generally, $a$ is larger at a location closer to the front corner of the T-junction than the one at a further downstream location. Consequently, Eq. (30) may be rewritten as

$$
|x| \approx \sqrt{\pi D_{C D} a t_{0}}, \quad 0<a<1 .
$$

Case 1 (see part I), for example, has a period of $7.8 \mathrm{~ms}$, and the maximum value of $a$ is approximated as $(7.8-1.6) / 7.8=$ 0.795 . Thus the maximum diffusion distance on the clear section under case 1 is about $5.4 \mu \mathrm{m}$ based on Eq. (32). It means that diffusion effect maximally covers a distance range from 0 to $5.4 \mu \mathrm{m}$ from the clear section of the interface under case 1 . This estimation results from the critical concentration $c_{x} \approx 0$; if $c_{x}=c_{0} / 2$ is applied, the corresponding distance range will be 0 to $2.7 \mu \mathrm{m}$. Regardless of the applied critical concentration, the diffusion distance $x$ compared with the channel width $W$ is a small value $(\leqslant 3.6 \%)$.

\section{MATHEMATICAL MODELS OF DROP LENGTH AND DROP SPACING}

In this section, mathematical descriptions of the drop length (normalized by the channel width) and the periodic development of the drop spacing are given based on our experimental results in part I. Some references to the concepts and parameters in part I may be necessary.

\section{A. Mathematical model of $\mathrm{L} / \mathrm{W}$ incorporating the "elongating" effect}

The normalized drop lengths (by channel width $W$ ) are correlated to the flow rate ratios $Q_{\mathrm{H}_{2} \mathrm{O}} / Q_{L \mathrm{CO}_{2}}$ in part I of our study. And the experimental results showed that the factor $A$ in Eq. (1) was much larger than 1, for the two groups of experiments, i.e., a constant total flow rate of $100 \mu \mathrm{l} / \mathrm{min}$ and a constant flow rate of the dispersed fluid (liquid $\mathrm{CO}_{2}$ ). On the other hand, factor $B$ in the scaling formulations were still in the vicinity of $(-1)$ which was consistent with the one reported by Garstecki et al. [1].

It is believed that the determination of $A$ in our study requires accounting for not only the "squeezing" (i.e., truncating) time but also the "elongating" time (see Fig. 2) when the liquid $\mathrm{CO}_{2}$ keeps entering and thus increases the length of the emerging drop, which is attributed to the elongatingsqueezing regime distinguished from the general "squeezing" regime. The final length $L$ of the drop is determined by how much $\mathrm{CO}_{2}$ has entered into the emerging drop in terms of length during the aforementioned three stages of one period, namely, the stagnating and filling stage, the elongating and squeezing stage, and the truncating stage (Fig. 2). Thus,

$$
L \sim\left(\Delta L_{\mathrm{sf}}+\Delta L_{\mathrm{es}}+\Delta L_{\mathrm{tr}}\right)
$$

(a)

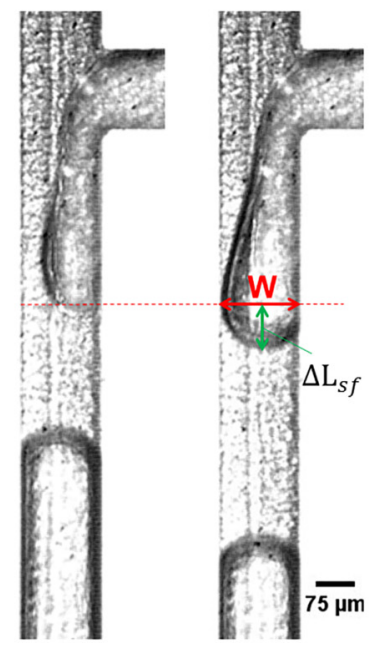

(b)

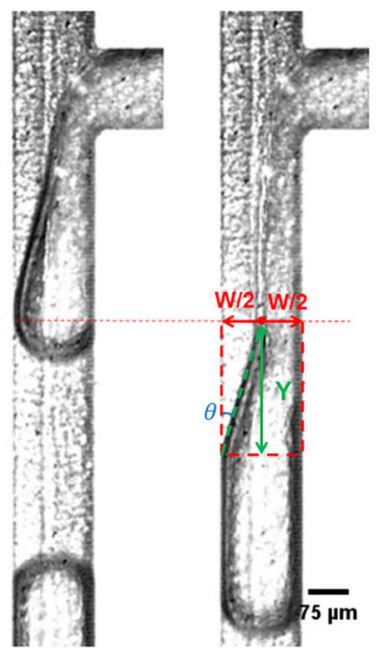

(c)

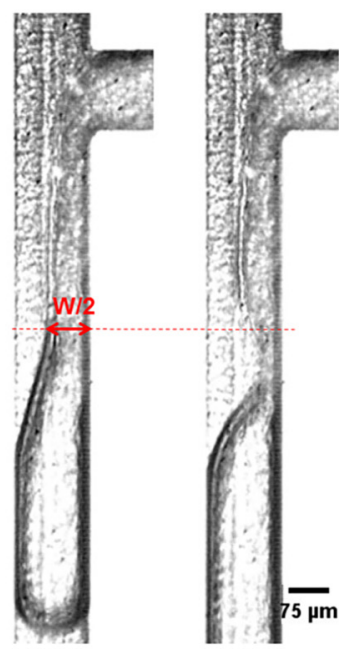

FIG. 2. Liquid $\mathrm{CO}_{2}$ drop length increases during the main three stages, namely, the stagnating and filling stage, the elongating and squeezing stage, and the truncating stage of one period of drop generation. (a) Drop length increase $\Delta L_{\mathrm{sf}}$ from the beginning to the end of the filling; (b) the time estimate of the elongating and squeezing stage by observing the advancing distance $(Y)$ of the water front from the filling end to the end of elongating and squeezing, the right frame shows that (1) the conjuncture between the clear and the shading section is located in the vicinity of the midpoint of the channel width and (2) the shading sectional line intersects the channel sideline with a characteristic angle $\theta$; (c) the truncating time estimate by considering the pinching off of the rest $W / 2$ thick $\mathrm{CO}_{2}$ stream. 
where $\Delta L_{\mathrm{sf}}, \Delta L_{\mathrm{es}}$, and $\Delta L_{\mathrm{tr}}$ are the drop length increases during the filling, elongating and squeezing, and truncating of the emerging drop, respectively. We adopt the notion that the length increase $\Delta L_{\mathrm{sf}}$ [Fig. 2(a)] as a result of $\mathrm{CO}_{2}$ filling (i.e., the length of the tip of the emerging drop) at the end of the first stage is on the order of channel width $W$ [1]. Therefore, we have

$$
\Delta L_{\mathrm{sf}} \sim W
$$

To determine $\Delta L_{\mathrm{es}}$, the time duration $t_{\mathrm{es}}$ of the elongating and squeezing stage needs to be estimated, which can be achieved by dividing the advancing distance of the continuous fluid (water) by its mean flow speed $Q_{\mathrm{H}_{2} \mathrm{O}} /(D W)$. It is observed that at the end of the elongating and squeezing stage [the right frame in Fig. 2(b)], for most of the investigated drop flow cases, the conjuncture between the clear section and inclined shading section at the interface is located nearby the centerline of the main channel; in addition, the two sectional interfacial lines approximate to straight lines and the shading section line intersect the sideline of the channel forming a characteristic angle $\theta$. The advancing distance $Y$ of water during this second stage can be calculated by

$$
Y=\frac{W / 2}{\tan \theta}
$$

and hence

$$
t_{\mathrm{es}} \sim \frac{Y}{Q_{\mathrm{H}_{2} \mathrm{O}} /(D W)}
$$

(a)

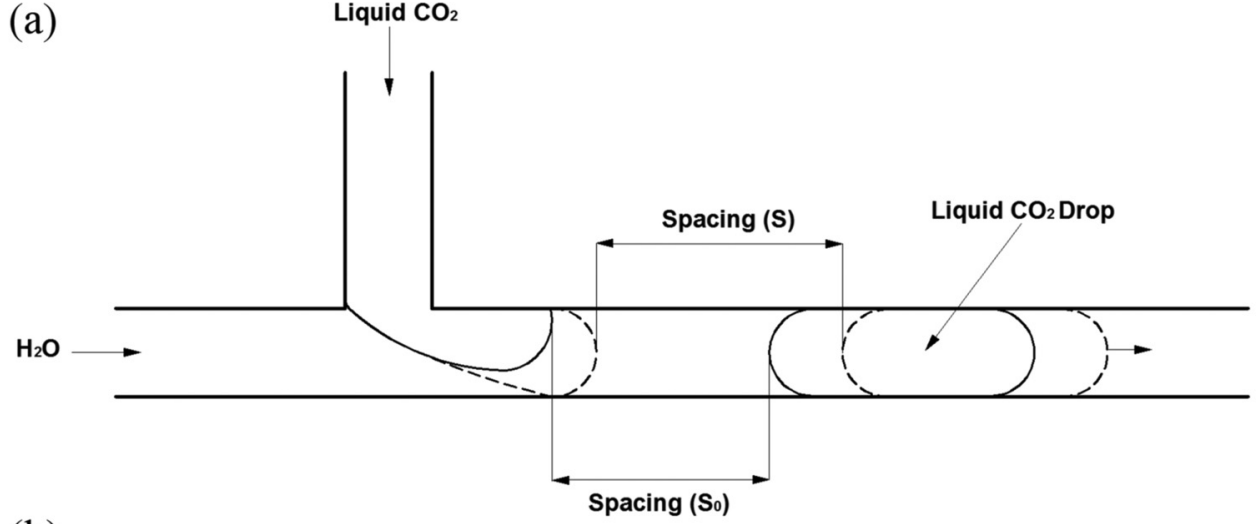

(b)

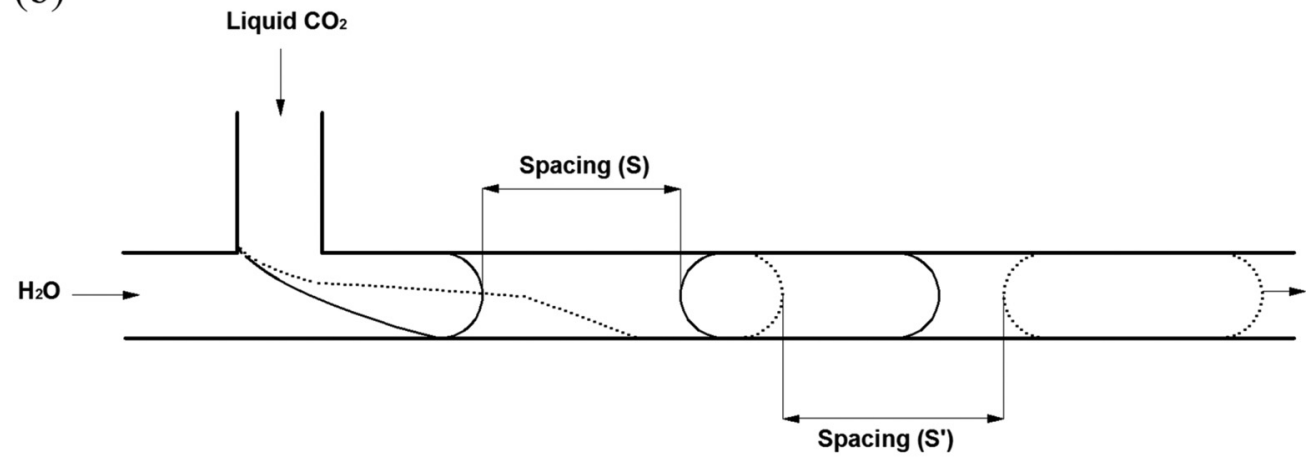

During the time, $t_{\mathrm{es}}$, liquid $\mathrm{CO}_{2}$ from the side channel keeps entering the emerging drop at a mean speed of $Q_{L \mathrm{CO}_{2}} /(D W)$. Based on these two parameters, the drop length increase, $\Delta L_{\mathrm{es}}$, can be estimated as

$$
\Delta L_{\mathrm{es}} \sim \frac{Y}{Q_{\mathrm{H}_{2} \mathrm{O}} /(D W)} \frac{Q_{L \mathrm{CO}_{2}}}{D W}=Y\left(Q_{\mathrm{H}_{2} \mathrm{O}} / Q_{L \mathrm{CO}_{2}}\right)^{-1} .
$$

Following the above strategy for $\Delta L_{\mathrm{es}}$, the drop length increase, $\Delta L_{\text {tr }}$, during the truncating stage can be estimated as

$$
\Delta L_{\mathrm{tr}} \sim \frac{W / 2}{Q_{\mathrm{H}_{2} \mathrm{O}} /(D W)} \frac{Q_{L \mathrm{CO}_{2}}}{D W}=\frac{W}{2}\left(Q_{\mathrm{H}_{2} \mathrm{O}} / Q_{L \mathrm{CO}_{2}}\right)^{-1} .
$$

Combining Eqs. (34), (37), and (38), the length $L$ of the generated liquid $\mathrm{CO}_{2}$ drop can be approximated as

$$
\begin{aligned}
L & \sim \Delta L_{\mathrm{sf}}+\Delta L_{\mathrm{es}}+\Delta L_{\mathrm{tr}} \\
& =W+Y\left(Q_{\mathrm{H}_{2} \mathrm{O}} / Q_{L \mathrm{CO}_{2}}\right)^{-1}+\frac{W}{2}\left(Q_{\mathrm{H}_{2} \mathrm{O}} / Q_{L \mathrm{CO}_{2}}\right)^{-1},
\end{aligned}
$$

and if the above equation is further divided by $W$ and rearranged, we obtain

$$
\frac{L}{W} \sim 1+\left(\frac{Y}{W}+\frac{1}{2}\right)\left(Q_{\mathrm{H}_{2} \mathrm{O}} / Q_{L \mathrm{CO}_{2}}\right)^{-1} .
$$

Substituting Eq. (35) into Eq. (40) yields

$$
\frac{L}{W} \sim 1+\left(\frac{1}{2 \tan \theta}+\frac{1}{2}\right)\left(Q_{\mathrm{H}_{2} \mathrm{O}} / Q_{L \mathrm{CO}_{2}}\right)^{-1} .
$$


By comparing Eq. (1) to Eq. (41), the factor $A$ corresponds to $\frac{1}{2}\left(\frac{1}{\tan \theta}+1\right)$. Given the value of $\theta\left[(12 \pm 1)^{\circ}\right]$ in our study, $A$ has a value of $(2.85 \pm 0.2)$ from the mathematical model, which agrees with the fitted values from our experimental data [see Figs. 7(a) and 7(b) in paper I].

\section{B. Mathematical model of periodic drop spacing $S$ between emerging drop and adjacent formed one}

We have reported the development of drop spacing within one period of the drop generation in the preceding paper. As shown by the representative drop flow cases (see Fig. 9 in paper I), the spacing always starts from an initial value $S_{0}$ and increases linearly during the two main stages (Fig. 3), i.e., the stagnating and filling stage and the elongating and squeezing stage, though the increasing rates vary due to narrowed drop speed difference over time.

During the filling stage prior to the emerging drop blocking the main channel, the already-formed drop moves downstream at a speed $\sim\left(V_{\mathrm{H}_{2} \mathrm{O}, a}+V_{\mathrm{CO}_{2}, a}\right)$ as discussed in the previous section; on the other hand, the emerging drop has a secondary effect "flowing downstream" other than filling, and its effective displacement is exactly the drop length increase $\Delta L(\sim W)$ during the filling time $t_{\mathrm{sf}}$, as a result, the effective mean speed of the emerging drop within the filling stage can be estimated as

$$
V_{\mathrm{sf}, \mathrm{eff}}=\Delta L_{\mathrm{sf}} / t_{\mathrm{sf}} \sim W / t_{\mathrm{sf}} .
$$

Based on the speed of the emerging drop and the already formed one together with the initial spacing $S_{0}$, the spacing in the filling stage increases linearly as a function of time, which can be formulated as

$$
S(t)=S_{0}+\left[\left(V_{\mathrm{CO}_{2}, a}+V_{\mathrm{H}_{2} \mathrm{O}, a}\right)-\frac{W}{t_{\mathrm{sf}}}\right](t-0), \quad 0<t \leqslant t_{\mathrm{sf}}
$$

or

$$
S(t)=S_{0}+\left(\frac{Q_{\mathrm{CO}_{2}}+Q_{\mathrm{H}_{2} \mathrm{O}}}{D W}-\frac{W}{t_{\mathrm{sf}}}\right) \cdot t, \quad 0<t \leqslant t_{\mathrm{sf}}
$$

Note that the estimate of spacing here may be applicable only to the drop flow cases excluding the dripping flows (case 27 and 28 in Fig. 5 in paper I) because either the speed of the emerging drop or that of the already formed drop needs to be amended considering that during the dripping regime, the emerging drop does not reach the far-end channel nor does $V_{\text {Total,a }}$ represent the actual averaged total velocity. However, the strategy for estimating spacing can be analogous for the dripping regime where the continuous fluid (specifically, its mean speed) may dominate the increasing rate of spacing [ $(S-$ $\left.\left.S_{0}\right) / t\right]$.

As soon as the emerging drop blocks the main channel, it commences the subsequent stages, and its mean speed at the front tip is supposed to be consisted of two components: one is contributed by the filling of liquid $\mathrm{CO}_{2}$ at a rate of $Q_{\mathrm{CO}_{2}}$ (i.e., $V_{\mathrm{CO}_{2}, a}$ ), and the other one by the continuous fluid via the contact upon the interface (more specifically, the shading section). As observed from the drop flows (excluding case
27 and 28), this latter speed component approximates to the moving speed of the inclined shading section of the interface [Fig. 2(b)], which is reasonable considering that the shading section is mainly propelled downstream by water especially under the drop flow cases resulted from low flow rate ratios. It is discovered that during the elongating and squeezing stage the water front in contact with the shading section advances downstream by a distance of $Y$ [Fig. 2(b)], and $Y$ can be approximated by $W /(2 \tan \theta)$. As a consequence, the mean speed of the shading section, $V_{\mathrm{sh}}$, over the time of the elongating and squeezing stage $\left(t_{\mathrm{es}}\right)$ plus that of the truncating stage $\left(t_{\mathrm{tr}}\right)$ can be determined as

$$
V_{\mathrm{sh}} \sim \frac{Y}{t_{\mathrm{es}}+t_{\mathrm{tr}}}
$$

Based on the values of $W$ and $\theta(W=150 \mu \mathrm{m}$ and $\theta \approx$ $\left.12^{\circ}\right), Y$ is calculated as a constant of $352.85 \mu \mathrm{m}$. Therefore, the mean speed $V_{\mathrm{sh}}$ becomes dependent on the total time $\left(t_{\mathrm{es}}+t_{\mathrm{tr}}\right)$ of the elongating and squeezing and the truncating stage. Moreover, the speed of the front tip of the emerging drop, $V_{\mathrm{CO}_{2}}$, tip , can be estimated by summing up $V_{\mathrm{CO}_{2}, a}$ and $V_{\text {sh }}$, such that

$$
V_{\mathrm{CO}_{2}, \text { tip }}=V_{\mathrm{CO}_{2}, a}+V_{\mathrm{sh}}
$$

or

$$
V_{\mathrm{CO}_{2}, \text { tip }}=\frac{Q_{\mathrm{CO}_{2}}}{D W}+\frac{Y}{t_{\mathrm{es}}+t_{\mathrm{tr}}} .
$$

Thus, the spacing increase $\left[S^{\prime}(t)-S\left(t_{\mathrm{sf}}\right)\right]$ during the elongating and squeezing and the truncating stage at the moment, $t$, can be calculated by

$$
\begin{aligned}
S^{\prime}(t)-S\left(t_{\mathrm{sf}}\right)= & {\left[\left(\frac{Q_{\mathrm{CO}_{2}}+Q_{\mathrm{H}_{2} \mathrm{O}}}{D W}\right)-\left(\frac{Q_{\mathrm{CO}_{2}}}{D W}+\frac{Y}{t_{\mathrm{es}}+t_{\mathrm{tr}}}\right)\right] } \\
& \times\left(t-t_{\mathrm{sf}}\right), t_{\mathrm{sf}}<t \leqslant t_{0},
\end{aligned}
$$

Substituting Eq. (44) at $t=t_{\text {sf }}$ into Eq. (48) and rearranging, we have

$$
\begin{aligned}
S^{\prime}(t)= & S_{0}+\left(\frac{Q_{\mathrm{CO}_{2}}+Q_{\mathrm{H}_{2} \mathrm{O}}}{D W}-\frac{W}{t_{\mathrm{sf}}}\right) \cdot t_{\mathrm{sf}} \\
& +\left(\frac{Q_{\mathrm{H}_{2} \mathrm{O}}}{D W}-\frac{Y}{t_{\mathrm{es}}+t_{\mathrm{tr}}}\right)\left(t-t_{\mathrm{sf}}\right), \quad t_{\mathrm{sf}}<t \leqslant t_{0} .
\end{aligned}
$$

Combining Eqs. (44) and (49), the spacing development over one period $t_{0}\left(t_{0}=t_{\mathrm{sf}}+t_{\mathrm{es}}+t_{\mathrm{tr}}\right)$ of drop generation can be described as

$$
\begin{aligned}
\text { spacing }= & S_{0}+\left(\frac{Q_{\mathrm{CO}_{2}}+Q_{\mathrm{H}_{2} \mathrm{O}}}{D W}-\frac{W}{t_{\mathrm{sf}}}\right) \cdot t, \quad 0<t \leqslant t_{\mathrm{sf}} \\
= & S_{0}+\left(\frac{Q_{\mathrm{CO}_{2}}+Q_{\mathrm{H}_{2} \mathrm{O}}}{D W}-\frac{W}{t_{\mathrm{sf}}}\right) \cdot t_{\mathrm{sf}} \\
& +\left(\frac{Q_{\mathrm{H}_{2} \mathrm{O}}}{D W}-\frac{Y}{t_{\mathrm{es}}+t_{\mathrm{tr}}}\right)\left(t-t_{\mathrm{sf}}\right), \quad t_{\mathrm{sf}}<t \leqslant t_{0} .
\end{aligned}
$$




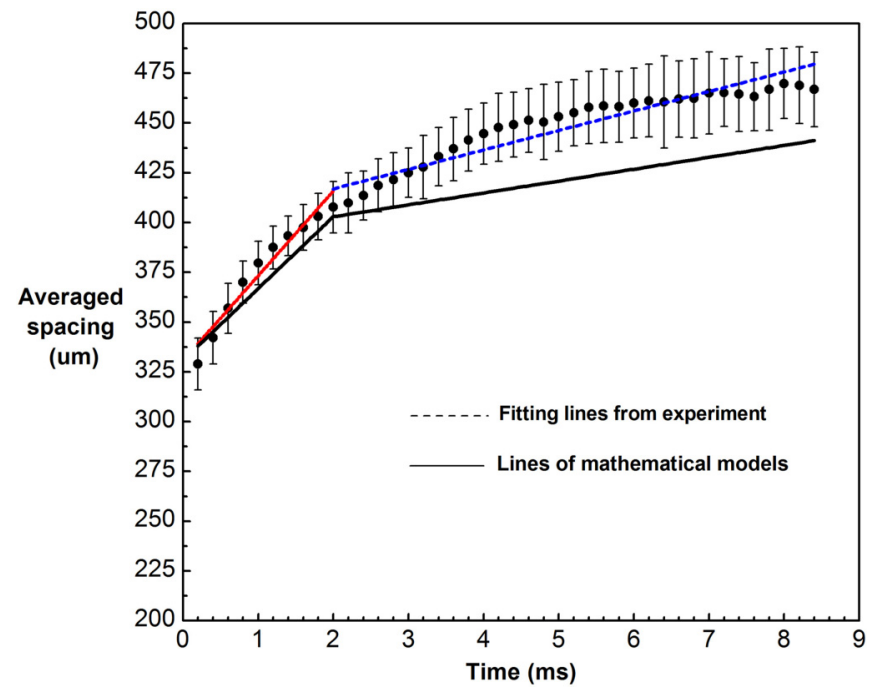

FIG. 4. Drop spacing development within one period $(8.4 \mathrm{~ms})$ of drop generation under drop flow case 14 . The experimental data $(\bullet)$ herein are averaged from those in the Fig. 9(b) in paper I, and each error bar indicates two standard deviations from the averaged spacing upon the corresponding time moment. Dashed lines are the fitting lines from the experimental data, and solid lines are the plots of the linear mathematical model in Eq. (50).

According to Eq. (50), the calculated spacing development within one period of the drop flow case 14 (which has been chosen as a model case in paper I) can be expressed as

$$
\begin{aligned}
\operatorname{spacing}(\mu \mathrm{m}) & =330.73+36.1 t, \quad 0<t \leqslant 2 \mathrm{~ms} \\
& =390.99+5.97 t, \quad 2<t \leqslant 8.4 \mathrm{~ms},
\end{aligned}
$$

which are also plotted in Fig. 4 for comparisons with those fitted from the experimental data. Note that the initial spacing, $S_{0}$, inherits from the first fitting above when $t=0$. Qualitatively, the theoretical model, Eq. (50), is consistent with the experimental fitting functions, which is a bit better during the first stage ( 0 to $2 \mathrm{~ms}$ ) of the one period $(8.4 \mathrm{~ms}$ ) but is slightly deviated for the elongating and squeezing and the truncating stage (2-8.4 ms). Moreover, the slopes in the models are both lower than those in the experimental fitting functions. These differences arise from the estimate of the total flow speed by using the total flow rate as well as a certain overestimate of the speed of the emerging drop. A quantitative comparison of the spacing between those resulted from the experimental fittings and the calculated ones from the mathematical model can be conducted as

$$
\begin{aligned}
\frac{\mid{\text { spacing }- \text { spacing }_{\text {ex }, \text { fit }} \mid}_{\text {spacing }_{\text {ex }, \text { fit }}}}{=} & \frac{6.45 t}{42.55 t+330.73}, \quad 0<t \leqslant 2 \mathrm{~ms} \\
& =\frac{3.81 t+6.36}{9.78 t+397.35}, 2<t \leqslant 8.4 \mathrm{~ms} .
\end{aligned}
$$

Therefore, the largest relative errors between the experimentally fitted spacing and the model predicted spacing are $3.1 \%$ at $t=2 \mathrm{~ms}$ for the stagnating and filling stage and $8 \%$ at $t=8.4 \mathrm{~ms}$ for the elongating and squeezing stage and the truncating stage, respectively. These errors are acceptable accounting for the uncertainty of the drop speed measurement under case 14 [see the Fig. 8(a) in paper I] where the mean drop speed is $114 \mathrm{~mm} / \mathrm{s}$ with a standard deviation of $6.5 \mathrm{~mm} / \mathrm{s}$.

\section{CONCLUSION}

In the second paper of a two-part series, we have provided theoretical justifications to our quantitative studies on the drop flow by considering the mass transport of the $\mathrm{CO}_{2}$ molecules driven by the diffusion controlled dissolution occurring in the vicinity of the interface. We have first evaluated the $\mathrm{CO}_{2}$ hydration at the interface with water assuming the interface reaches a saturation scenario of $\mathrm{CO}_{2}$ dissolution. The hydration shows an overall negligible effect to the total dissolved $\mathrm{CO}_{2}$ molecules in terms of amount and the vast majority of the dissolved $\mathrm{CO}_{2}$ molecules still present in the aqueous state. Second, we have estimated the diffusion-controlled dissolution of $\mathrm{CO}_{2}$ molecules in water based on a hydrostatic assumption and found that a saturation scenario of the dissolved $\mathrm{CO}_{2}$ molecules in the vicinity of the interface would not be reached within the contacting time. The dissolution section was followed by an analysis on the diffusion distance of the dissolved $\mathrm{CO}_{2}$ molecules. Molecular diffusion does play a role in transferring the dissolved $\mathrm{CO}_{2}$ molecules, but the diffusion distance (or "diffusive thickness") is very limited compared with the channel geometry.

In addition, mathematical models for the drop length and the drop spacing have been developed based on the observations in paper I and are compared to our experimental results. We have reported a characteristic interface angle $\theta$ formed by the channel wall and the interface at the end of the elongating and squeezing stage that commenced the truncation of the dispersed stream. This angle was applied to accomplish our model of the drop length, and the factor $A$ in the model was successfully quantified, which agrees with that resulted from the experimental fittings. Moreover, based on the speed differences between the emerging drop and the adjacent newly generated one, a linear mathematical model was developed to predict the drop spacing as a function of the time for, respectively, addressing the stagnating and filling stage and the elongating-squeezing stage of the emerging drop generation. In view of a periodic occurrence of the spacing development, the model agrees well with experimental results and the relative errors are reasonable.

\section{ACKNOWLEDGMENTS}

N.Q. gratefully acknowledges the financial support from the China Scholarship Council (CSC) under File No. 201204910160. The authors acknowledge the Carbon Management Canada (Theme C: Secure Carbon Storage, Project C393) and the Networks of Centres of Excellence (NCE) of Canada for research grants. 
[1] P. Garstecki, M. J. Fuerstman, H. A. Stone, and G. M Whitesides, Lab. Chip. 6, 437 (2006).

[2] M. De Menech, P. Garstecki, F. Jousse, and H. A. Stone, J. Fluid Mech. 595, 141 (2008).

[3] D. M. Anderson, G. B. McFadden, and A. A. Wheeler, Annu. Rev. Fluid Mech. 30, 139 (1998).

[4] M. Abolhasani, M. Singh, E. Kumacheva, and A. Gunther, Lab. Chip. 12, 1611 (2012).

[5] T. Cubaud, M. Sauzade, and R. P. Sun, Biomicrofluidics 6, 022002 (2012).

[6] C. E. Housecroft and A. G. Sharpe, Inorganic Chemistry (Pearson Prentice Hall, Harlow, England, 2005).

[7] K. S. Johnson, Limnol. Oceanogr. 27, 849 (1982).

[8] K. G. Schulz, U. Riebesell, B. Rost, S. Thoms, and R. E. Zeebe, Mar. Chem. 100, 53 (2006).

[9] N. N. Greenwood and A. Earnshaw, Chemistry of the Elements (Butterworth-Heinemann, Oxford, 1997).

[10] A. V. Bandura and S. N. Lvov, J. Phys. Chem. Ref. Data 35, 15 (2006).
[11] A. Einstein, Ann. Phys. 322, 549 (1905).

[12] J. R. Li, Y. G. Ma, M. C. McCarthy, J. Sculley, J. M. Yu, H. K. Jeong, P. B. Balbuena, and H. C. Zhou, Coordin. Chem. Rev. 255, 1791 (2011).

[13] N. Spycher, K. Pruess, and J. Ennis-King, Geochim. Cosmochim. Ac. 67, 3015 (2003).

[14] L. W. Diamond and N. N. Akinfiev, Fluid Phase Equilibr. 208, 265 (2003).

[15] K. Pruess and K. Zhang, Numerical modeling studies of the dissolution-diffusion-convection process during $\mathrm{CO}_{2}$ storage in saline aquifers, Lawrence Berkeley National Laboratory, 2008.

[16] A. Fick, Ann. Phys. 170, 59 (1855).

[17] H. S. Carslaw and J. C. Jaeger, Conduction of Heat in Solids (Oxford University Press, Oxford, 1986).

[18] N. Perez, Electrochemistry and Corrosion Science (Kluwer Academic Publishers, Norwell, MA, 2004). 